OPEN ACCESS

Edited by:

Yanjie Wei,

Shenzhen Institutes of Advanced Technology (CAS), China

Reviewed by:

Quan Zou,

University of Electronic Science and Technology of China, China

Fei Guo,

Tianjin University, China

*Correspondence:

Tao Huang

tohuangtao@126.com

Yu-Dong Cai

cai_yud@126.com

tThese authors have contributed equally to this work

Specialty section:

This article was submitted to

Molecular Diagnostics and Therapeutics,

a section of the journa

Frontiers in Molecular Biosciences

Received: 10 September 2020

Accepted: 15 October 2020

Published: 04 November 2020

Citation:

Chen L, Li Z, Zeng T, Zhang Y-H,

Liu D, Li H, Huang T and Cai Y-D

(2020) Identifying Robust Microbiota

Signatures and Interpretable Rules

to Distinguish Cancer Subtypes.

Front. Mol. Biosci. 7:604794.

doi: 10.3389/fmolb.2020.604794

\section{Identifying Robust Microbiota Signatures and Interpretable Rules to Distinguish Cancer Subtypes}

\author{
Lei Chen ${ }^{1,2+}$, Zhandong Lijit, Tao Zeng ${ }^{4}$, Yu-Hang Zhang ${ }^{5}$, Dejing Liu', Hao $\mathrm{Li}^{3}$, \\ Tao Huang ${ }^{6 *}$ and Yu-Dong Cai ${ }^{*}$ \\ ${ }^{1}$ School of Life Sciences, Shanghai University, Shanghai, China, ${ }^{2}$ College of Information Engineering, Shanghai Maritime \\ University, Shanghai, China, ${ }^{3}$ College of Food Engineering, Jilin Engineering Normal University, Changchun, China, \\ ${ }^{4}$ Zhangjiang Laboratory, Institute of Brain-Intelligence Technology, Shanghai, China, ${ }^{5}$ Channing Division of Network \\ Medicine, Brigham and Women's Hospital, Harvard Medical School, Boston, MA, United States, ${ }^{6}$ Key Laboratory of Tissue \\ Microenvironment and Tumor, Shanghai Institute of Nutrition and Health, Chinese Academy of Sciences, Shanghai, China
}

Cancer can be generally defined as a cluster of systematic diseases triggered by abnormal cell proliferation and growth. With the development of biological sciences and biotechnologies, the etiology of cancer is partially revealed, including some of the most substantial pathogenic factors [either endogenous (genetics) or exogenous (environmental)]. However, some remaining factors that contribute to the tumorigenesis but have not been analyzed and discussed in detail remain. For instance, some typical correlations between microorganisms and tumorigenesis have been reported already, but previous studies are just sporadic studies on single microorganism-cancer subtype pairs and do not explain and validate the specific contribution of microbiome on tumorigenesis. On the basis of the systematic microbiome analyses of blood and cancer-associated tissues in cancer patients/controls in public domain, we performed interpretable analyses. We identified several core regulatory microorganisms that contribute to the classification of multiple tumor subtypes and established quantitative predictive models for interpretable prediction by using multiple machine learning methods. We also compared the optimal features (microorganisms) and rules identified from microbiome profiles processed using the Kraken and the SHOGUN. Collectively, our study identified new microbiome signatures and their interpretable classification rules for cancer discrimination and carried out reliable methodological comparison for robust cancer microbiome analyses, thereby promoting the development of tumor etiology at the microbiome level.

Keywords: cancer type, microbiota, machine learning algorithm, decision tree, rules

\section{INTRODUCTION}

Cancer, as one of the most threatening diseases all over the world, can be generally defined as a cluster of systematic diseases triggered by abnormal cell proliferation and growth (McGuire, 2016; Vanhoutte et al., 2016). According to the World Health Organization (Vanhoutte et al., 2016; Shams-White et al., 2019), cancer is the second leading cause of death compared with other diseases and causes nearly 10 million deaths and about 20 million newly reported cases worldwide 
in 2018. China has comparable cancer morbidity and a relatively quite high mortality with the world average, and about 4 million new cancer cases and 3 million cancer-associated deaths are reported in 2018 from China (Feng et al., 2019; Shams-White et al., 2019), indicating that cancer is one of the most threatening diseases in China.

With the development of biological sciences and the progress of biotechnologies, the etiology of cancer is partially revealed, including some of the most significant pathogenic factors [either endogenous (genetics) or exogenous (environmental)]. In previous studies, the endogenous [like genes EGFR (Wang et al., 2019), TP53 (Salk et al., 2019), and RAS (Lanfredini et al., 2019)] and the exogenous [like smoking (Croyle et al., 2019), alcoholism (Srivastava et al., 2019) and severe air pollutions (Guo et al., 2019)] factors are widely reported to participate in tumorassociated biological processes, some of which are reported to directly trigger the initiation of tumorigenesis. Current studies are systematic and thorough, but some remaining factors that contribute to the tumorigenesis but are not analyzed and discussed in detail remain.

The relationships between microorganisms and cancers have been reported for decades. For viruses, in the seventh decade of the 20th century, the infection of the hepatitis $B$ virus (HBV) is correlated with the initiation and the progression of hepatocellular carcinoma after a long course of HBV infections (Feng et al., 2019). Two types of human papillomavirus, i.e., HPV16 and HPV-18, are identified as the most important pathogens for cervical cancers (Shibata et al., 2019). Vaccines against HPV16 and HPV-18 are developed and promoted among adolescent girls and adult women to prevent the high incidence of cervical cancers (Di Bonito et al., 2019; Shibata et al., 2019). Apart from the virus, some bacteria are functionally correlated with certain cancer subtypes. For instance, Helicobacter pylori, as a digestive infection bacteria, is reported to promote the initiation and the progression of gastric cancers (Mentis et al., 2019). Although some typical correlations between microorganisms and tumorigenesis are already reported, previous studies are just sporadic studies on single microorganism-cancer subtype pairs but do not explain and validate the specific contribution of microbiome on tumorigenesis.

In March, 2020, a systematic microbiome analyses of blood and cancer-associated tissues in cancer patients/controls reflect the characteristic distribution of the microbiome among different cancer subtypes and their potential contributions to the tumorigenesis procedures (Poore et al., 2020). For the first time, such research has identified some typical signatures of multiple cancer subtypes and tried to identify specific biomarkers with diagnostic or prediction potentials on cancer, confirming that different cancer subtypes have different microbiome profiling patterns. Some optimal biomarkers (microorganisms) from either tumor or blood can be applied for the early diagnosis of certain cancer subtypes. In this study, on the basis of the initial microbiome analyses results, we have further performed two levels of interpretable analyses. On the one hand, we have identified some core regulatory microorganisms that contribute to the classification of multiple tumor subtypes and established quantitative predictive models for accurate prediction by using multiple machine learning methods. On the other hand, we have performed and compared the optimal features (microorganisms) and rules identified from two microbiome profiles [i.e., processed using the Kraken (Wood et al., 2019) and the SHOGUN (Hillmann et al., 2020)] by considering the original study that applied two major sequencing and analysis workflows. Overall, our study has identified new biomarkers and their interpretable classification rules for cancer microbiome discrimination by relying on the systematic analysis of microbiome profiling data and compared the Kraken and the SHOGUN methods for robust cancer microbiome analyses, promoting the development of tumor etiology at microbiome level.

\section{MATERIALS AND METHODS}

\section{Data}

We downloaded the processed microbiome data of TCGA patients with cancer from ftp://ftp.microbio.me/pub/cancer_ microbiome_analysis/(Poore et al., 2020). The data were processed using two different methods, i.e., the Kraken (Wood and Salzberg, 2014) and the SHOGUN (Hillmann et al., 2018). Therefore, two datasets were generated. They all relies on sequence alignment and reference-based taxonomy annotation to identify potential microorganisms from the microbiome data.

The Kraken method can be sequentially divided into three major steps (Wood and Salzberg, 2014):

(1) Mapping k-mers of the query sequence to references of multiple taxonomy.

(2) Identifying all the taxonomies that contain high quality mapped sequences.

(3) Building a weighted classification tree and find the path from root (high level classification category) to leaf (low level classification category) with the highest added score. And the leaf in the classification path with the highest added score is the classification used for the query sequence. The Kraken can also be described as using the $\mathrm{k}$-mers of each sequence to find the lowest common ancestor (LCA) as the final annotation.

As for the SHOGUN method, it can also be divided into three major steps (Hillmann et al., 2018):

(1) Using three methods (Bowtie2, BURST, and UTree) to align the candidate sequence to the genome.

(2) Using weighted last-common ancestor algorithm to annotate each sequence with one taxonomy with confidence generated from all the mapped reads. Further, the BURST aligner can help build a rank-specific relative profiling, finding the most relative profiling of such candidate sequence.

(3) The SHOGUN also applies Bracken algorithm to estimate rank-specific relative abundance using each genome's uniqueness, profiling hits number and length.

There are two major similarities and three differences between such two computational methods (Wood and Salzberg, 2014; Hillmann et al., 2018). 
The similarities include:

(1) The initial step of both methods is mapping to the candidate genomes of multiple microorganisms.

(2) Both methods try to assign one unique taxonomy to each sequence to avoid redundant annotation.

The differences include:

(1) SHOGUN method takes the coverage of target reference and abundance characteristics of the query sequence into consideration to calculate the confidence of annotated taxonomies, while Kraken only control the mapping procedure using aligners' degree of confidence.

(2) Both methods try to annotate the sequence using one taxonomy, but using different methods: UTree in SHOGUN trying to find "the lowest-common-ancestor scheme" for annotation, while Kraken has its own scoring methods, which search for the root-to-leaf path with the lowest score, taking the entire classification path into consideration not just the final ancestor.

(3) SHOGUN can also evaluate relative abundance of each candidate annotated taxonomies, while Kraken cannot.

As we have presented above, both methods were solid microorganisms identification methods. Considering the differences of such two methods, it is quite reasonable and acceptable for us to identify some different candidate microbiomes for our further classification analyses.

In the Kraken dataset, 17,625 microbiomes in 1993 samples were obtained from 32 cancer types. In the SHOGUN dataset, 13,517 microbiomes in 1594 samples were obtained from 32 cancer types. We believed that different cancers had different microbiomes, indicating cancer-specific microbiomes. The sample sizes of each cancer type in the Kraken and the SHOGUN datasets are shown in Table 1. Features used to represent samples in each dataset were different. Figure 1 shows the number of common and different features in each dataset. Evidently, each dataset contained several exclusive features. An analysis on these two datasets can give a complete view of different cancer types with microbiome.

\section{Minimum Redundancy Maximum Relevance (mRMR)}

The mRMR (Peng et al., 2005) is a powerful and widely used feature selection method. The informative features evaluated by such method should have (i) minimum redundancy among themselves and (ii) maximum relevance with class labels. To this end, the method employed mutual information (MI) to evaluate the relationships between features or class labels. For two variables $x$ and $y$, their MI value can be formulated by

$$
I(x, y)=\iint p(x, y) \log \frac{p(x, y)}{p(x) p(y)} d x d y
$$

where $p(x) / p(y)$ stands for the marginal probabilistic density of the variable and $p(x, y)$ indicates the joint probabilistic density of two variables. The mRMR aims to evaluate the importance of features in a list, simultaneously satisfying the above two points. Initially, such list is empty. The feature is selected from the rest features one by one, which has maximum relevance with class labels and minimum redundancy to features already in the list. When all features are in the list, the entire procedures stop. For convenience, the obtained feature list was denoted by $F$ in this study.

This study used the mRMR program retrieved from http://penglab.janelia.org/proj/mRMR/. Default parameters were adopted.

\section{Incremental Feature Selection (IFS)}

Although the mRMR method can sort the features with the decreasing order of their importance, it is still difficult to determine which features are essential. This study employed the IFS (Liu and Setiono, 1998) method, which can be used

TABLE 1 | Summary of the Kraken and SHOGUN datasets.

\begin{tabular}{|c|c|c|c|}
\hline \multirow[t]{2}{*}{ Index } & \multirow[t]{2}{*}{ Cancer Type } & \multicolumn{2}{|c|}{ Sample size } \\
\hline & & $\begin{array}{l}\text { Kraken } \\
\text { dataset }\end{array}$ & $\begin{array}{l}\text { SHOGUN } \\
\text { dataset }\end{array}$ \\
\hline 1 & Adrenocortical carcinoma & 79 & 79 \\
\hline 2 & Bladder urothelial carcinoma & 729 & 729 \\
\hline 3 & Brain lower grade glioma & 731 & 731 \\
\hline 4 & Breast invasive carcinoma & 1483 & 1483 \\
\hline 5 & $\begin{array}{l}\text { Cervical squamous cell carcinoma and } \\
\text { endocervical adenocarcinoma }\end{array}$ & 451 & 451 \\
\hline 6 & Cholangiocarcinoma & 45 & 45 \\
\hline 7 & Colon adenocarcinoma & 1006 & 417 \\
\hline 8 & Esophageal carcinoma & 340 & 340 \\
\hline 9 & Glioblastoma multiforme & 489 & 338 \\
\hline 10 & Head and Neck squamous cell carcinoma & 906 & 297 \\
\hline 11 & Kidney chromophobe & 191 & 65 \\
\hline 12 & Kidney renal clear cell carcinoma & 1141 & 1114 \\
\hline 13 & Kidney renal papillary cell carcinoma & 393 & 23 \\
\hline 14 & Liver hepatocellular carcinoma & 523 & 162 \\
\hline 15 & Lung adenocarcinoma & 911 & 911 \\
\hline 16 & Lung squamous cell carcinoma & 638 & 534 \\
\hline 17 & $\begin{array}{l}\text { Lymphoid neoplasm diffuse large b-cell } \\
\text { lymphoma }\end{array}$ & 61 & 61 \\
\hline 18 & Mesothelioma & 87 & 87 \\
\hline 19 & Ovarian serous cystadenocarcinoma & 1031 & 1031 \\
\hline 20 & Pancreatic adenocarcinoma & 183 & 183 \\
\hline 21 & Pheochromocytoma and Paraganglioma & 186 & 186 \\
\hline 22 & Prostate adenocarcinoma & 829 & 829 \\
\hline 23 & Rectum adenocarcinoma & 372 & 372 \\
\hline 24 & Sarcoma & 347 & 347 \\
\hline 25 & Skin cutaneous melanoma & 792 & 667 \\
\hline 26 & Stomach adenocarcinoma & 1079 & 1079 \\
\hline 27 & Testicular germ cell tumors & 139 & 139 \\
\hline 28 & Thymoma & 122 & 122 \\
\hline 29 & Thyroid carcinoma & 880 & 287 \\
\hline 30 & Uterine carcinosarcoma & 57 & 57 \\
\hline 31 & Uterine corpus endometrial carcinoma & 1222 & 169 \\
\hline 32 & Uveal melanoma & 182 & 182 \\
\hline
\end{tabular}


TABLE 2 | Summary of the performance of the best model with different classification algorithms on two datasets.

\begin{tabular}{|c|c|c|c|c|c|c|}
\hline \multirow[t]{2}{*}{ Classification algorithm } & \multicolumn{3}{|c|}{ Kraken dataset } & \multicolumn{3}{|c|}{ SHOGUN dataset } \\
\hline & Number of features & ACC & MCC & Number of features & ACC & MCC \\
\hline Random forest & 582 & 0.921 & 0.918 & 146 & 0.884 & 0.878 \\
\hline Support vector machine & 1989 & 0.588 & 0.575 & 1592 & 0.633 & 0.616 \\
\hline k-nearest neighbor & 682 & 0.812 & 0.804 & 277 & 0.895 & 0.889 \\
\hline Decision tree & 580 & 0.736 & 0.724 & 1481 & 0.824 & 0.814 \\
\hline
\end{tabular}

to determine the best number of essential features for a given classification algorithm. At first, IFS produced a series of feature subsets from the above-constructed feature list $F$. For example, the first feature subset consisted of one top-ranked feature, and the second feature subset consisted of two top-ranked features, and so forth. Then, for each feature subset, the IFS trained a classifier on the training samples with features in such set. And this classifier was evaluated by 10 -fold cross-validation (Kohavi, 1995). Finally, the IFS determined the optimum feature subset, on which the classification model provided the best performance evaluated by Matthew correlation coefficient (MCC) (Matthews, 1975).

\section{Synthetic Minority Oversampling Technique (SMOTE)}

As listed in Table 1, two microbiome datasets had different numbers of samples in different cancer types. For the Kraken dataset, the largest cancer type had about 33 times samples as many as the smallest type, whereas for the SHOGUN, this number was about 64.5. It is indicated that these two datasets were imbalanced. To reduce the influence of the imbalance, SMOTE (Chawla et al., 2002) was adopted when evaluating the performance of each classification model. This method produces new samples for the minor sample class, thereby ensuring that the number of samples in the minor class was equivalent to that of samples in the major class after an iterative procedure. In detail, it randomly selects a sample, say $x$, in the minor class and finds out some nearest samples to it in the same class. Then, randomly pick up a nearest sample, say $y$, among above nearest samples.

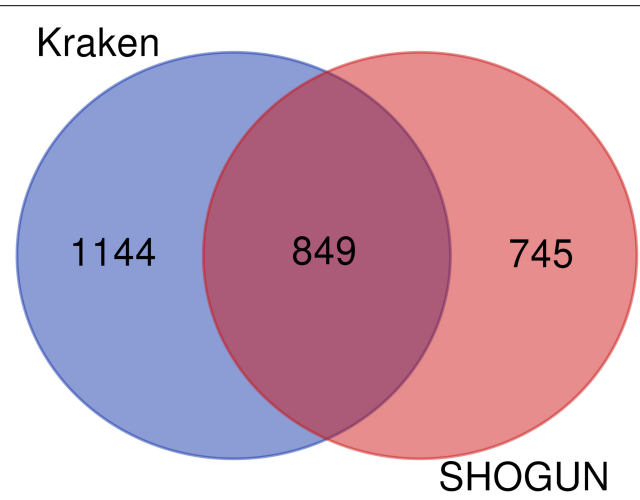

FIGURE 1 | Venn diagram to show the common and different features used in two datasets. Several exclusive features are contained in each dataset.
A new sample is generated by a linear combination of $x$ and $y$. Because the new generated sample has strong associations with $x$ and $y$, it has a high probability to be in the same class of $x$ and $y$. Thus, it is also assigned such class label. In this study, the SMOTE was employed to enlarge each cancer type except the largest one. Finally, each type has equal number of samples. The "SMOTE" tool available in Weka (Witten and Frank, 2005) was applied in this work.

\section{Classification Algorithm}

Four classification models were used in the microbiome feature learning and rule extraction.

\section{Random Forest (RF)}

The RF (Breiman, 2001; Wei et al., 2017; Zhao et al., 2018; Baranwal et al., 2019; Jia et al., 2020; Liang et al., 2020) is a treebased assembly model that predicts the class label of a new sample on the basis of the consensus results of the average predictions from multiple decision trees (DTs). In the present study, we used the RF implemented in the Scikit-learn package.

\section{Support Vector Machine (SVM)}

The SVM (Cortes and Vapnik, 1995; Sun et al., 2015; Chen et al., 2017; Sang et al., 2020; Zhou et al., 2020a,b) can transform the data point from a low-dimensional data space to a highdimensional data space. The SVM divides the data samples of each label in the principle of data interval maximization in a high-dimensional space and predicts the class label of a new sample depending on the interval to which this new sample belongs. The SMO algorithm in the Weka software is used to build the SVM model.

\section{k-Nearest Neighbor (kNN)}

The kNN (Cover and Hart, 1967) first calculates the distance between the test and the training samples and ranks the training samples by using their distance from the test sample. The kNN then selects the $k$ high-ranked training samples (i.e., nearest neighbors), estimates the label distribution of such $k$ samples, and predicts the label of the test sample as the class label with the highest frequency of the label distribution. The IBk algorithm in the Weka software is used to build the kNN model.

\section{DT}

The DT (Safavian and Landgrebe, 1991) aims to build the human understanding classification and the regression models by using interpretative rules in a white box model, e.g., using the IF-TEHN format to describe individual features roles and weights in the 
classification and the regression models. The CART algorithm with the Gini index in the Scikit-learn package was used to build the DT model.

\section{Performance Evaluation}

The MCC (Matthews, 1975), which can evaluate the performance of the classification model, has values from -1 to +1 and achieves +1 when one classification model has the best performance. In this work, the multiclass version of the MCC (Gorodkin, 2004) was applied because the analyzed microbiome data were organized as multiple categories and can be calculated as:

$$
M C C=\frac{\operatorname{cov}(X, Y)}{\sqrt{\operatorname{cov}(X, X) \operatorname{cov}(Y, Y)}},
$$

where the binary matrix $X$ indicates the predicted class of each sample, the binary matrix $Y$ represents the true classes of all samples, and $\operatorname{cov}(X, Y)$ represents the covariance of two matrices.

\section{RESULTS}

In this study, we analyzed two microbiome datasets using several computational methods. The entire procedures are illustrated in Figure 2.

\section{mRMR Results}

The Kraken and the SHOGUN datasets were all analyzed by the mRMR method. As a result, two feature lists were produced, which are available in Supplementary Tables S1, S2, respectively.

\section{IFS Results}

The feature lists were obtained by applying mRMR method to the Kraken and the SHOGUN datasets, which were fed into the IFS with four classification algorithms.

Of the feature list on the Kraken dataset, we constructed several models with one classification algorithm and some top features in the list. Each model was assessed by 10 -fold crossvalidation. Obtained measurements, including accuracy on each cancer type, overall accuracy (ACC) and MCC, are provided in Supplementary Table S3. For an easy observation, an IFS curve was plotted for each classification algorithm with MCC as the $Y$-axis and number of features as the $X$-axis, which is illustrated in Figure 3. When RF was selected as the classification algorithm, the highest MCC was 0.918. It was obtained based on the top 582 features. The highest MCCs for the other three algorithms were $0.804,0.724$, and 0.575 , respectively, which were based on top 682, 580, and 1989 features. These highest MCCs and corresponding ACCs were collected in Table 2. Evidently, the RF with top 582 features was the best model among all tested models. In addition, the accuracies on 32 cancer types yielded by above best models with different classification algorithms are shown in Figure 4A. Clearly, The RF model yielded higher accuracies on cancer types than those obtained by other models. We called the 582 features used in such RF model as the global optimum features on the Kraken dataset.

For the feature list on the SHOGUN dataset, same procedures were done. The performance of all tested models is listed in

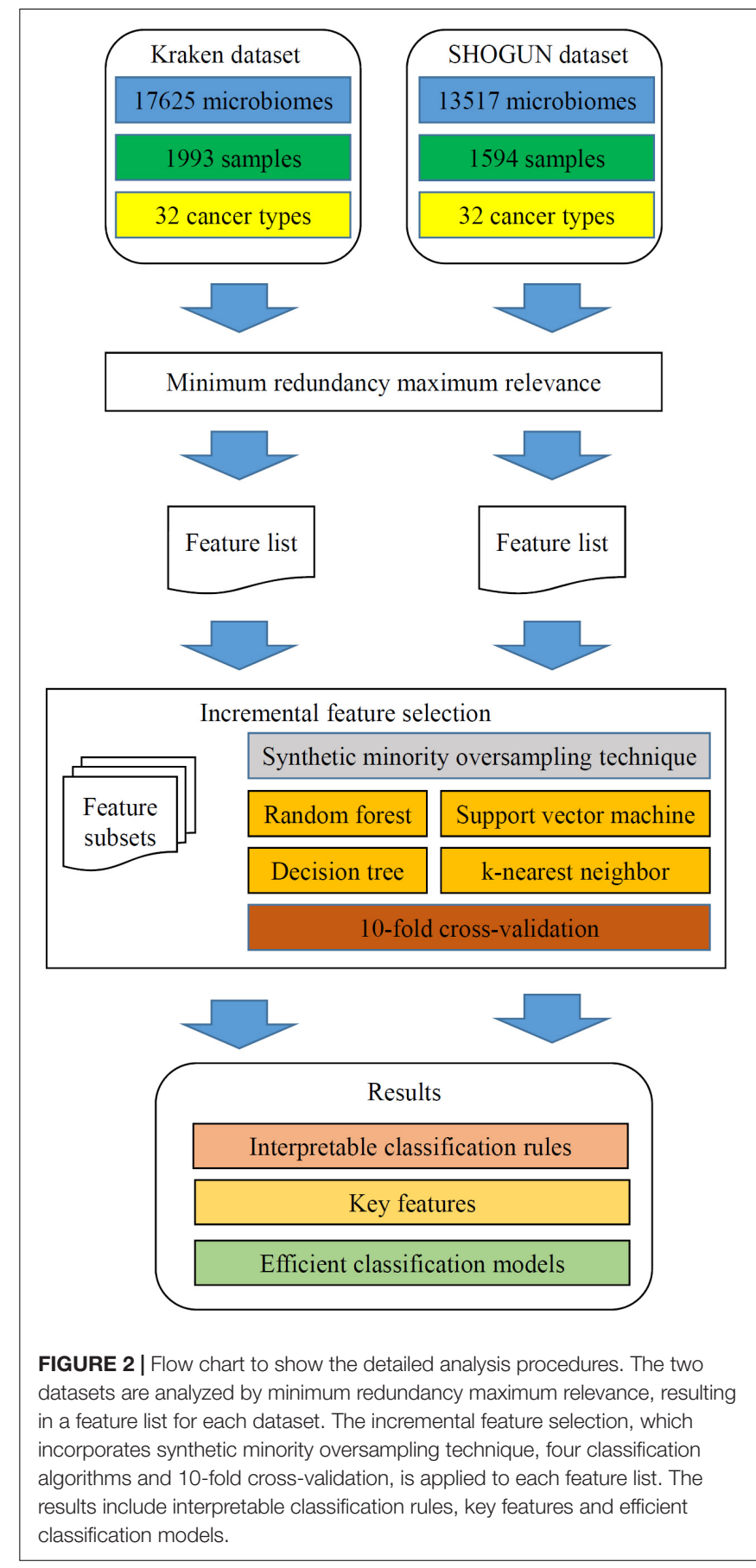

Supplementary Table S4. Also, four IFS curves were plotted, as shown in Figure 5. It can be observed that the highest MCCs for different classification algorithms were $0.889,0.878,0.814$, and 0.616 , respectively, which were based on top $277,146,1481$, and 1592 features, respectively. Above MCCs and corresponding ACCs are listed in Table 2. Among these best models with different classification algorithms, the kNN with top 277 features was the best. To further confirm this fact, the accuracies on 32 cancer types yielded by the best models using different classification algorithms are shown in Figure 4B. Evidently, the 


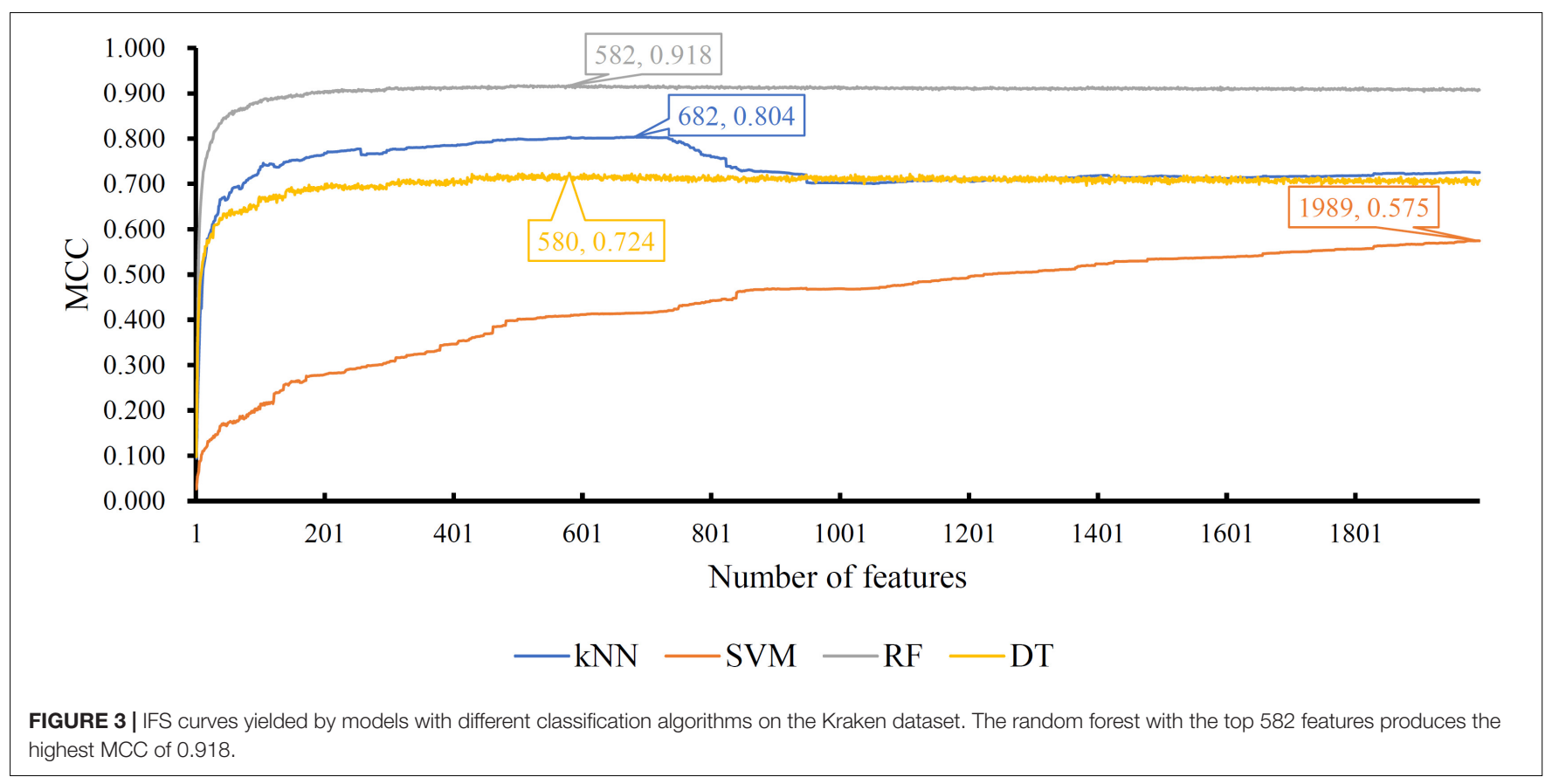

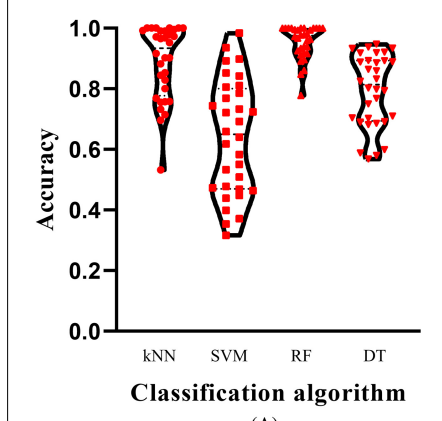

(A)

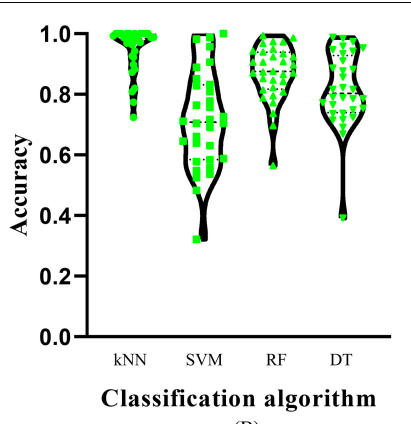

(B)
FIGURE 4 | Violin plot to show the accuracies on cancer types which are produced by the best model with different classification algorithms on two datasets. (A) Kraken dataset, the RF model yields the most high accuracies; (B) SHOGUN dataset, the kNN model produces the most high accuracies.

accuracies produced by the kNN model were in higher levels than those produced by other models. Accordingly, these 277 features were called global optimum features on the SHOGUN dataset.

Given a classification algorithm, IFS method can detect its optimum features on each dataset. In detail, for RF, 582 and 146 optimum features were extracted from Kraken and SHOGUN datasets, respectively. A Venn diagram was plotted to show the common and difference of these two feature sets (Figure 6A), from which we can see that several exclusive features were extracted from each dataset. Similar situations occurred for other three classification algorithms (see Figures 6B-D). Besides, we also analyzed the common and difference of the global optimum features on two datasets (Figure 7). Also, several exclusive features were obtained for each dataset. Above results indicated that the two datasets can provide different information on cancer type at microbiome level. Analyzing them together can give a more complete view on such problem.

\section{Classification Rules}

As mentioned in section "IFS Results," DT can provide the highest MCC on the Kraken dataset when top 580 features were used. Thus, we applied DT on the Kraken dataset, in which samples were represented by these features. As a result, 3579 rules were obtained, which are provided in Supplementary Table S5. Each cancer type was related to some rules. Rules (310) on cancer type "Breast Invasive Carcinoma" were most, while those (10) on "Uterine Carcinosarcoma" were least. The number of rules related to each cancer type is listed in column 3 of Table 3.

Of the SHOGUN dataset, DT with top 1481 features was best. Accordingly, we applied DT on this dataset, where samples were represented by these 1481 features. As a result, 2030 rules were accessed. These rules are available in Supplementary Table S6. In such rule group, rules (173) on cancer type "Breast Invasive Carcinoma" were still most, while rules (5) on cancer type "Uveal Melanoma" were least. The number of rules related to each cancer type is listed in column 4 of Table 3.

Detailed investigation on above rules can improve our understanding on different cancer types at microbiome level. Some rules were analyzed in section "Discussion."

\section{DISCUSSION}

Here, we have identified the essential microorganisms for the distinction of different tumor subtypes on the basis of the optimal features produced from two microbiome computational methods (i.e., the Kraken and the SHOGUN). According to recent publications, all predicted microorganisms with distinctive 


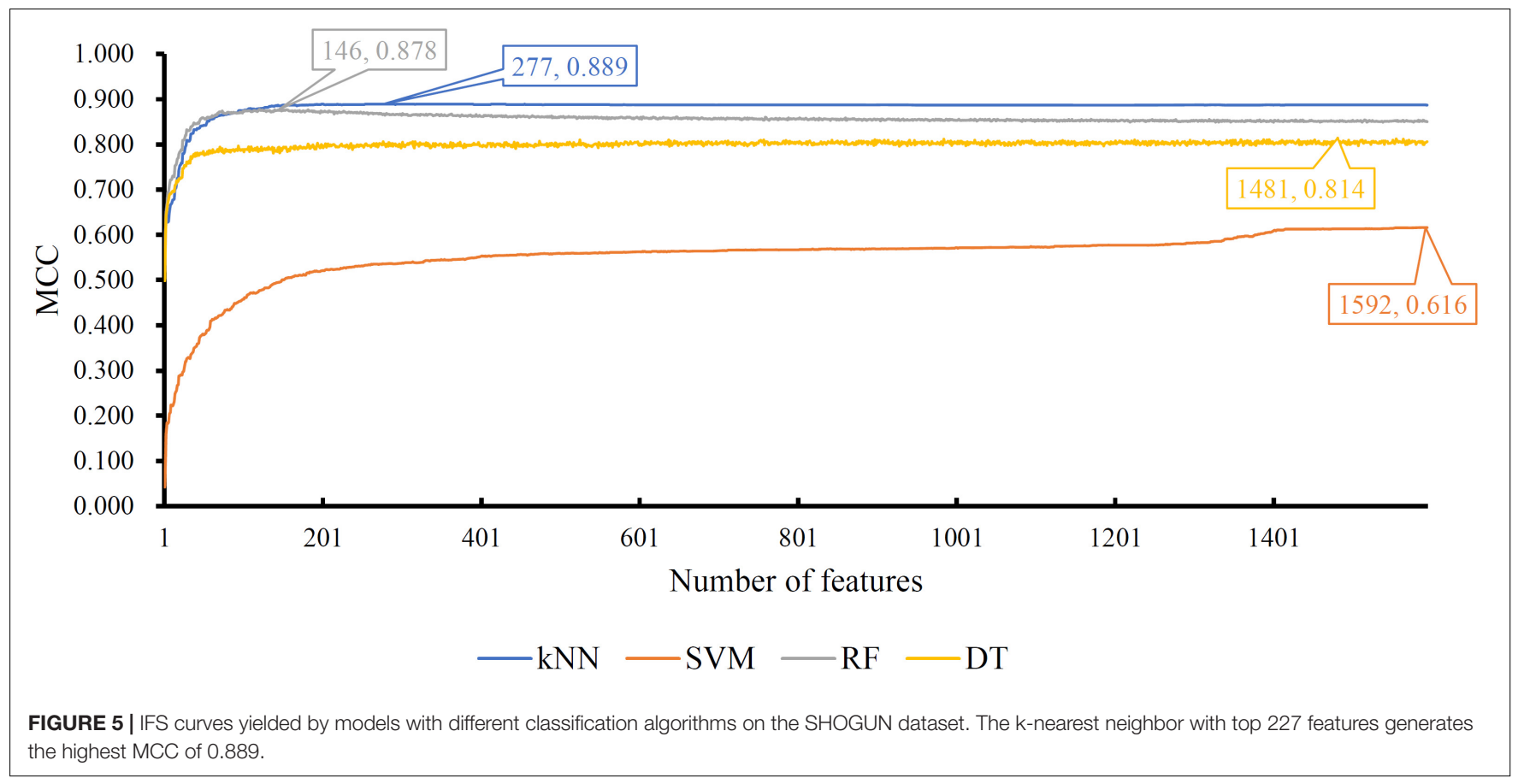

FIGURE 5 | IFS curves yielded by models with different classification algorithms on the SHOGUN dataset. The k-nearest neighbor with top 227 features generates
the highest MCC of 0.889 .
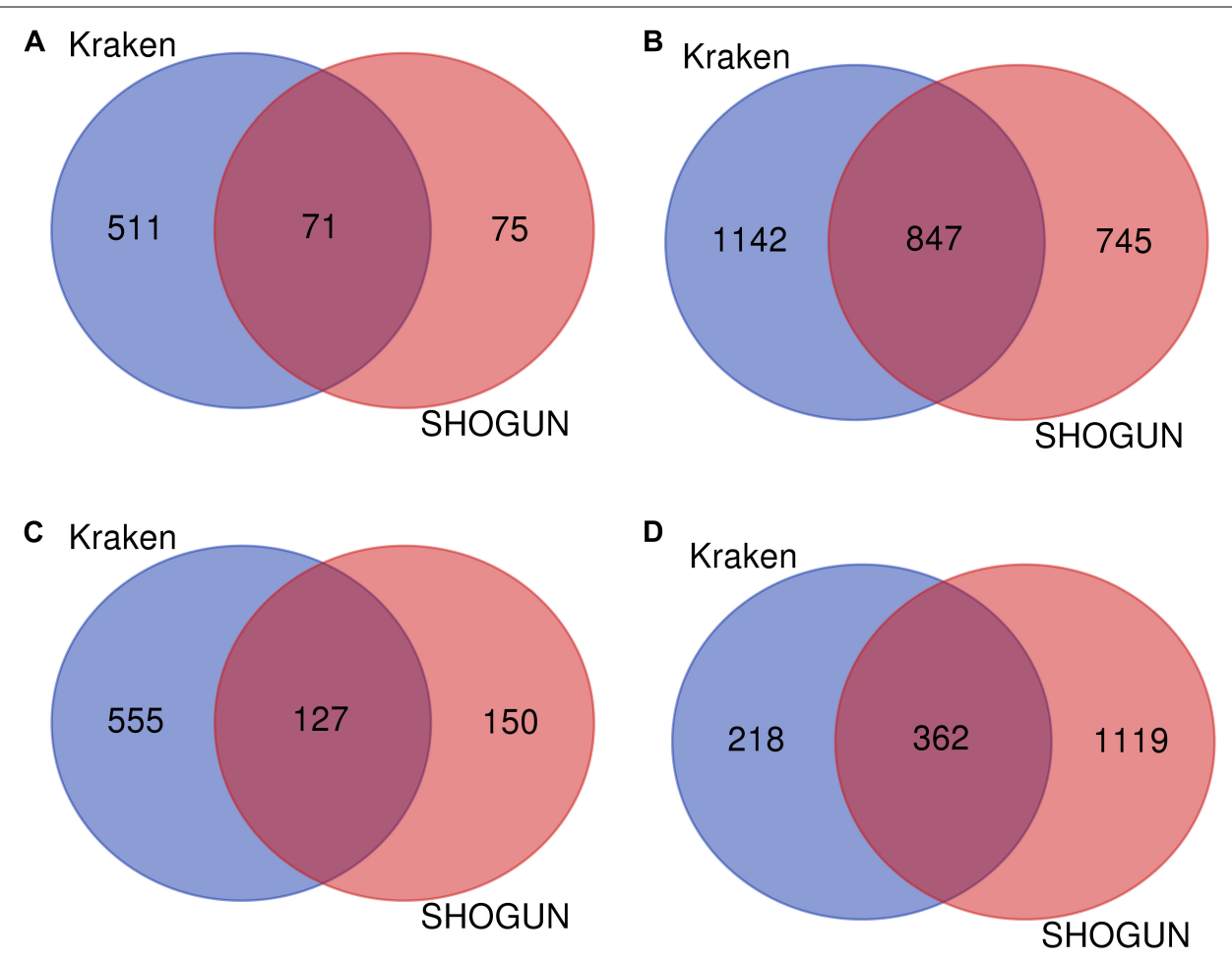

FIGURE 6 | Venn diagram to show the common and difference of optimum feature subsets by applying a given classification algorithm to each of two datasets. (A) Random forest; (B) Support vector machine; (C) k-nearest neighbor; (D) Decision tree.

capacity are validated and functionally correlated with one or multiple tumor subtypes. Apart from such qualitative analysis to identify potential tumor subtyping signatures, we have further built up a group of quantitative rules for detailed tumor classification, and these rules are also supported by related literature and datasets in the public domain. The detailed analyses on the optimal features (microorganisms) and their rules can be seen below. 


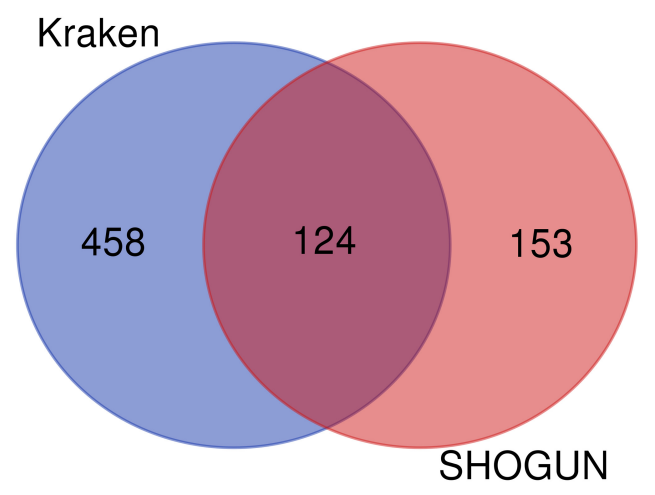

FIGURE 7 | Venn diagram to show the common and difference of the global optimum feature subsets on two datasets.

\section{Key Features Identified From the Microbiome Data Produced by the Kraken Method}

The first microorganism in our prediction list on the Kraken data is Robiginitomaculum from the specific genus named Hyphomonadaceae. According to recent publications, a research preprint from the bioRxiv has confirmed that such microorganism may share some similar sequences with the Sonic hedgehog factors in multiple animals and contribute to the internal regulation of related signaling pathways due to such sequence similarities (Jägers and Roelink, 2019). Sonic hedgehog factors are key regulators for the hedgehog signaling pathway, which has been widely reported to contribute to multiple cancer subtypes, including basal cell carcinomas (Daya-Grosjean and Couvé-Privat, 2005), prostate cancer (Datta and Datta, 2006), and pancreatic cancer (Nakashima et al., 2006) with different expression profiling. Therefore, such bacteria can contribute to the detailed classification of multiple cancer subtypes, thereby validating the efficacy and the accuracy of our prediction.

The next predicted microorganism, i.e., Mycoplasma, is a specific kind of microorganism without cell wall around the cell membrane. Mycoplasma, as a unique kind of microorganism, is reported to be correlated with multiple cancer subtypes with infections detected either in blood [like cervical cancer (Zhu et al., 2007)] or tumor in situ [like prostate (Barykova et al., 2011), gastric (Yang et al., 2010), and ovarian (Chan et al., 1996) cancers]. Furthermore, the detailed mechanisms for Mycoplasma contributing to tumorigenesis are reported. The microorganism can directly cause pathological chromosomal loss and translocations in multiple cell subtypes (Chan et al., 1996).

The third tumor-associated pathogen, Lachnoclostridium, is predicted to be functionally correlated with tumorigenesis and may further participate in the detailed tumor classification. According to recent publications, as one of the most famous member of the gut microbiome, these bacteria are functionally correlated with colorectal adenoma and cancer (Liang et al., 2020). Another independent study further validates that such
TABLE 3 | Number of rules for each cancer type on two datasets.

\begin{tabular}{|c|c|c|c|}
\hline \multirow[t]{2}{*}{ Index } & \multirow[t]{2}{*}{ Cancer Type } & \multicolumn{2}{|c|}{ Number of rules } \\
\hline & & $\begin{array}{l}\text { Kraken } \\
\text { dataset }\end{array}$ & $\begin{array}{l}\text { SHOGUN } \\
\text { dataset }\end{array}$ \\
\hline 1 & Adrenocortical carcinoma & 24 & 8 \\
\hline 2 & Bladder urothelial carcinoma & 235 & 168 \\
\hline 3 & Brain lower grade glioma & 128 & 53 \\
\hline 4 & Breast Invasive carcinoma & 310 & 173 \\
\hline 5 & $\begin{array}{l}\text { Cervical squamous cell carcinoma and } \\
\text { endocervical adenocarcinoma }\end{array}$ & 91 & 89 \\
\hline 6 & Cholangiocarcinoma & 12 & 13 \\
\hline 7 & Colon adenocarcinoma & 217 & 112 \\
\hline 8 & Esophageal carcinoma & 55 & 33 \\
\hline 9 & Glioblastoma multiforme & 31 & 18 \\
\hline 10 & Head and Neck squamous cell carcinoma & 228 & 85 \\
\hline 11 & Kidney chromophobe & 40 & 16 \\
\hline 12 & Kidney renal clear cell carcinoma & 164 & 104 \\
\hline 13 & Kidney renal papillary cell carcinoma & 132 & 22 \\
\hline 14 & Liver hepatocellular carcinoma & 122 & 60 \\
\hline 15 & Lung adenocarcinoma & 232 & 150 \\
\hline 16 & Lung squamous cell carcinoma & 143 & 135 \\
\hline 17 & $\begin{array}{l}\text { Lymphoid neoplasm diffuse large b-cell } \\
\text { lymphoma }\end{array}$ & 15 & 18 \\
\hline 18 & Mesothelioma & 19 & 32 \\
\hline 19 & Ovarian serous cystadenocarcinoma & 59 & 35 \\
\hline 20 & Pancreatic adenocarcinoma & 65 & 59 \\
\hline 21 & Pheochromocytoma and Paraganglioma & 26 & 29 \\
\hline 22 & Prostate adenocarcinoma & 203 & 137 \\
\hline 23 & Rectum adenocarcinoma & 89 & 86 \\
\hline 24 & Sarcoma & 56 & 68 \\
\hline 25 & Skin cutaneous melanoma & 210 & 94 \\
\hline 26 & Stomach adenocarcinoma & 129 & 69 \\
\hline 27 & Testicular germ cell tumors & 38 & 23 \\
\hline 28 & Thymoma & 33 & 14 \\
\hline 29 & Thyroid carcinoma & 199 & 101 \\
\hline 30 & Uterine carcinosarcoma & 10 & 10 \\
\hline 31 & Uterine corpus endometrial carcinoma & 228 & 11 \\
\hline 32 & Uveal melanoma & 36 & 5 \\
\hline Total & - & 3579 & 2030 \\
\hline
\end{tabular}

microorganism may even contribute to the non-invasive detection of colorectal cancer (Mangifesta et al., 2018), implying that such bacteria can act as an effective classification parameter to identify colorectal cancers from other cancer subtypes.

Achromobacter and Acidithiobacillus are the next two predicted microorganisms identified on the Kraken data and predicted to be essential classification parameters by our newly presented computational methods. As a pathogen for respiratory tract infection, Achromobacter is reported to be correlated with multiple cancer subtypes related to the respiratory tract (Barragán et al., 2018; Nolley et al., 2019), confirming its potential contribution on cancer subtyping. Similarly, Acidithiobacillus infects lung cells and contributes to the initiation of lung cancer (Ramírez-Aldaba et al., 2017) but not to other cancer subtypes. 


\section{Rules Identified From the Microbiome Data Produced by the Kraken Method}

Apart from such qualitative analyses on the mapping and annotation results following the Kraken data, we have identified some quantitative rules for the identification of certain cancer subtypes.

Among such rules, a specific rule contributing to the identification of breast cancer is established with multiple quantitative parameters, including Succinimonas and Campylobacter. These two microorganisms are chosen as typical parameters for detailed discussion. The microorganism named Succinimonas is functionally correlated with the metabolism of breast lactation in cows and human beings (Elolimy et al., 2018). Considering that the breast lactation metabolism is correlated with breast cancer tumorigenesis (Kim and Wysolmerski, 2016; Wani et al., 2017), this microorganism is regarded as a potential quantitative parameter for breast cancer. Many studies have identified the infection of Campylobacter in breast cancer (Korneev et al., 2018; Parida and Sharma, 2019), further validating the efficacy and the accuracy of our prediction.

Apart from breast cancer, the urothelial bladder carcinoma is identified using multiple rules. Among them, in a typical rule, Acidibacillus and Nitrospira are two typical microorganisms that may contribute to the tumorigenesis of such cancer subtype and may participate in the distinction from other cancer subtypes. According to recent publications, these two microorganisms are identified in biological samples from patients with urothelial bladder carcinoma, indicating that both microorganisms are enriched in urothelial bladder carcinoma-associated tissues (Oliveira, 2014; Weng et al., 2016).

Some specific quantitative parameters are screened for sarcoma. Collinsella and Hepacivirus are identified to contribute to the progression of such disease. In 2019, Collinsella is reported as one of the most important gut microbiota that contribute to the initiation and the progression of sarcoma, and these findings correspond with our prediction rules (Vivarelli et al., 2019). As for Hepacivirus, according to recent publications, Kaposi's sarcoma is functionally correlated with the hepatitis $\mathrm{C}$ virus, validating our prediction on the upregulated level of Hepacivirus in such tumor subgroup (Ray et al., 1995; Wu et al., 2018).

\section{Key Features Identified From the Microbiome Data Produced by the SHOGUN Method}

A similar analysis is performed on the SHOGUN data, and the first microorganism in our prediction result is Caballeronia, which is widely shown to be functionally correlated with the biosynthesis of D-tagatose ( $\mathrm{Li}$ et al., 2019). The intake and the metabolism of D-tagatose in human beings are reported to be functionally correlated with the specific cell cycle arrest in hepatocytes (Yamaguchi et al., 2008), which contribute to the initiation and the progression of hepatocellular carcinoma. Therefore, such microorganism may be applied to distinguish hepatocellular carcinoma from other cancer subtypes.
The next predicted microorganism can be classified into the Gammaproteobacteria (order). As for its distinctive contribution on different cancer subtypes with specific distribution patterns in human beings, such microorganism has been identified in the pathogenic tissues of two specific digestive systemassociated cancer subtypes, i.e., colorectal (Peters et al., 2016) and pancreatic (Choy et al., 2018) cancers.

Another predicted microorganism named as Chlamydia is similar with Mycoplasma as we have analyzed above and a typical subtype of prokaryotic organisms with severe pathogenic capacity. According to recent publications, such organisms are identified in multiple cancer subtypes, including cervical cancer with reproductive tract Chlamydia infection (Koskela et al., 2000; Smith et al., 2004) and lung cancer with respiratory tract Chlamydia infection (Laurila et al., 1997; Littman et al., 2005). Such microorganism can infect exposed mucosal tissues and induce tumorigenesis at the regional infection sites, implying its potential capacity on distinguishing different tumor subtypes via their relationship with mucosal tissues.

Moreover, the predicted microorganism named as Bradyrhizobium has quite few reports on its differential correlations with different cancer subtypes (only reported to be detected in the serum samples from cancer patients) (Nordlund et al., 2005). The predicted microorganism named as Kurthia participates in the malignant tumorigenesis, and its distribution may be functionally correlated with the colorectal cancer tumorigenesis. The abundance profiling of such microorganism in gut may contribute to the diagnosis and the prognosis prediction on colorectal cancer at least in a mouse model (Yu et al., 2018).

\section{Rules Identified From the Microbiome Data Produced by the SHOGUN Method}

Apart from such microorganisms predicted as qualitative parameters for cancer subtyping on the SHOGUN data, we have established some effective prediction rules for the accurate prediction of certain cancer subtypes on the basis of the detailed microorganism abundance. The optimal rules and the associated features are discussed below.

Specific rules for ovarian cancers are established. The high abundances of Oribacterium and Selenomonas are predicted to be correlated with the initiation and the progression of ovarian cancers. For Oribacterium, recent publications have confirmed that such microorganism is functionally correlated with the hemorrhagic ovarian cyst syndrome in the pathological ovary (Thackray, 2019). Considering that the hemorrhagic ovarian cyst syndrome is one of the typical precancerous lesions of ovarian cancer, speculating the tight correlations between Oribacterium and ovarian cancers is quite reasonable (Brown and Frumovitz, 2014). Selenomonas is confirmed to be correlated with multiple cancer subtypes, including oral and ovarian (Al-Hebshi et al., 2019) cancers, and these findings correspond with our rules.

Typical rules contributing to the identification of kidney renal clear cell carcinoma are also identified. Among them, two typical parameters are named as Terasakiispira and Candidatus. Thiodiazotropha contributes to the identification 
of such cancer subtype with publication support. Terasakiispira is detected in the pathological urinary system, including the malignant transformed urinary system (Schultz et al., 2020), and contributes to abnormal genomic alterations in human beings (Angiuoli et al., 2008).

As the final examples, specific rules about the uterine carcinosarcoma involve multiple parameters, including Natronococcus and Terasakiispira. Both microorganisms are functionally correlated with tumorigenesis (Angiuoli et al., 2008; Hamidi et al., 2019). Although the lack of direct connections between such two microorganisms with the uterine carcinosarcoma, their upregulation at least confirms the malignant transformation in candidate tissues, validating our prediction.

\section{Comparison of Features and Rules Identified Between the Kraken and the SHOGUN Methods}

The results obtained from two kinds of data are compared. Among 1993 Kraken- and 1594 SHOGUN-based predicted microorganisms, 907 specific species are identified to be shared in both methods, implying the reproducibility and the comparability of the two analytic microbiome methods and validating the efficacy and the accuracy of our new prediction methods. For top predicted microorganisms, the detailed species name may vary, but multiple genera are identified to be shared in the 20 top-ranked microorganisms, like Mycoplasmataceae, Enterococcaceae, and Rhodobacteraceae, which indeed have solid publication support to be correlated with the tumorigenesis of some cancer subtypes.

\section{CONCLUSION}

Overall, the optimal features and rules in our prediction lists have been validated by recent publications, and they are robust and efficient for different analytic microbiome methods (i.e., Kraken and SHOGUN). Our study has identified a group of novel potential biomarkers/rules for the subgrouping of different cancer subtypes on the microbiome level and provided an effective computational tool to identify the potential associations between microbiome and tumorigenesis, thereby exploring the complicated microenvironment components associated with tumorigenesis.

\section{REFERENCES}

Al-Hebshi, N. N., Borgnakke, W. S., and Johnson, N. W. (2019). The microbiome of oral squamous cell carcinomas: a functional perspective. Curr. Oral Health Rep. 6, 145-160. doi: 10.1007/s40496-019-0215-5

Angiuoli, S. V., Gussman, A., Klimke, W., Cochrane, G., Field, D., Garrity, G., et al. (2008). Toward an online repository of Standard Operating Procedures (SOPs) for (meta)genomic annotation. OMICS 12, 137-141. doi: 10.1089/omi.2008. 0017

Baranwal, M., Magner, A., Elvati, P., Saldinger, J., Violi, A., and Hero, A. O. (2019). A deep learning architecture for metabolic pathway prediction. Bioinformatics 36, 2547-2553. doi: 10.1093/bioinformatics/btz954

\section{DATA AVAILABILITY STATEMENT}

Publicly available datasets were analyzed in this study. This data can be found here: ftp://ftp.microbio.me/pub/cancer microbiome_analysis/.

\section{AUTHOR CONTRIBUTIONS}

TH and Y-DC designed the study. LC, ZL, TZ, and Y-HZ performed the experiments. LC, ZL, DL, and HL analyzed the results. LC and ZL wrote the manuscript. All authors contributed to the research and reviewed the manuscript.

\section{FUNDING}

This work was supported by the Shanghai Municipal Science and Technology Major Project (2017SHZDZX01), National Key R\&D Program of China (2018YFC0910403), National Natural Science Foundation of China (31701151), Shanghai Sailing Program (16YF1413800), Youth Innovation Promotion Association of Chinese Academy of Sciences (CAS) (2016245), and Fund of the Key Laboratory of Tissue Microenvironment and Tumor of Chinese Academy of Sciences (202002).

\section{SUPPLEMENTARY MATERIAL}

The Supplementary Material for this article can be found online at: https://www.frontiersin.org/articles/10.3389/fmolb. 2020.604794/full\#supplementary-material

Supplementary Table S1 | Rank of relevant features from mRMR on
the Kraken dataset.

Supplementary Table S2 | Rank of relevant features from mRMR on the SHOGUN dataset.

Supplementary Table S3 | Performance of IFS with different classification models under different numbers of features on the Kraken dataset.

Supplementary Table S4 | Performance of IFS with different classification models under different numbers of features on the SHOGUN dataset.

Supplementary Table S5 | Rules generated by decision tree on the Kraken dataset.

Supplementary Table $\mathbf{S 6}$ | Rules generated by decision tree on the SHOGUN dataset.

Barragán, E. P., Pérez, J. S., Corbella, L., Orellana, M., and FernándezRuiz, M. (2018). Achromobacter xylosoxidans bacteremia: clinical and microbiological features in a 10-year case series. Rev. Español. Quimioter. 31:268.

Barykova, Y. A., Logunov, D. Y., Shmarov, M. M., Vinarov, A. Z., Fiev, D. N., Vinarova, N. A., et al. (2011). Association of Mycoplasma hominis infection with prostate cancer. Oncotarget 2:289. doi: 10.18632/oncotarget.256

Breiman, L. (2001). Random forests. Mach. Learn. 45, 5-32. doi: 10.1023/A: 1010933404324

Brown, J., and Frumovitz, M. (2014). Mucinous tumors of the ovary: current thoughts on diagnosis and management. Curr. Oncol. Rep. 16:389. doi: 10.1007/ s11912-014-0389-x 
Chan, P. J., Seraj, I. M., Kalugdan, T. H., and King, A. (1996). Prevalence of mycoplasma conserved DNA in malignant ovarian cancer detected using sensitive PCR-ELISA. Gynecol. Oncol. 63, 258-260. doi: 10.1006/gyno.1996. 0316

Chawla, N. V., Bowyer, K. W., Hall, L. O., and Kegelmeyer, W. P. (2002). SMOTE: synthetic minority over-sampling technique. J. Artif. Intell. Res. 16, 321-357. doi: 10.1613 /jair.953

Chen, L., Wang, S., Zhang, Y.-H., Li, J., Xing, Z.-H., Yang, J., et al. (2017). Identify key sequence features to improve CRISPR sgRNA efficacy. IEEE Access 5, 26582-26590. doi: 10.1109/access.2017.2775703

Choy, A. T., Carnevale, I., Coppola, S., Meijer, L. L., Kazemier, G., Zaura, E., et al. (2018). The microbiome of pancreatic cancer: from molecular diagnostics to new therapeutic approaches to overcome chemoresistance caused by metabolic inactivation of gemcitabine. Exp. Rev. Mol. Diagnost. 18, 1005-1009. doi: 10. 1080/14737159.2018.1544495

Cortes, C., and Vapnik, V. (1995). Support-vector networks. Mach. Learn. 20, 273-297. doi: 10.1007/BF00994018

Cover, T., and Hart, P. (1967). Nearest neighbor pattern classification. IEEE Trans. Inf. Theory 13, 21-27. doi: 10.1109/TIT.1967.1053964

Croyle, R., Morgan, G., and Fiore, M. (2019). Addressing a core gap in cancer care: the NCI Cancer MoonshotSM initiative to help oncology patients stop smoking. New Engl. J. Med. 380:512. doi: 10.1056/nejmp1813913

Datta, S., and Datta, M. (2006). Sonic Hedgehog signaling in advanced prostate cancer. Cell. Mol. Life Sci. 63, 435-448. doi: 10.1007/s00018-0055389-4

Daya-Grosjean, L., and Couvé-Privat, S. (2005). Sonic hedgehog signaling in basal cell carcinomas. Cancer Lett. 225, 181-192. doi: 10.1016/j.canlet.2004. 10.003

Di Bonito, P., Accardi, L., Galati, L., Ferrantelli, F., and Federico, M. (2019). Anticancer vaccine for HPV-associated neoplasms: focus on a therapeutic HPV vaccine based on a novel tumor antigen delivery method using endogenously engineered exosomes. Cancers 11:138. doi: 10.3390/cancers 11020138

Elolimy, A. A., Abdelmegeid, M. K., Mccann, J. C., Shike, D. W., and Loor, J. J. (2018). Residual feed intake in beef cattle and its association with carcass traits, ruminal solid-fraction bacteria, and epithelium gene expression. J. Anim. Sci. Biotechnol. 9:67. doi: 10.1186/s40104-018-0283-8

Feng, J., Yang, G., Liu, Y., Gao, Y., Zhao, M., Bu, Y., et al. (2019). LncRNA PCNAP1 modulates hepatitis B virus replication and enhances tumor growth of liver cancer. Theranostics 9:5227. doi: 10.7150/thno.34273

Feng, R.-M., Zong, Y.-N., Cao, S.-M., and Xu, R.-H. (2019). Current cancer situation in China: good or bad news from the 2018 global cancer statistics? Cancer Commun. 39:22. doi: 10.1186/s40880-019-0368-6

Gorodkin, J. (2004). Comparing two K-category assignments by a K-category correlation coefficient. Comp. Biol. Chem. 28, 367-374. doi: 10.1016/j. compbiolchem.2004.09.006

Guo, H., Chang, Z., Wu, J., and Li, W. (2019). Air pollution and lung cancer incidence in China: who are faced with a greater effect? Environ. Int. 132:105077. doi: 10.1016/j.envint.2019.105077

Hamidi, M., Mirzaei, R., Delattre, C., Khanaki, K., Pierre, G., Gardarin, C., et al. (2019). Characterization of a new exopolysaccharide produced by Halorubrum sp. TBZ112 and evaluation of its anti-proliferative effect on gastric cancer cells. 3 Biotech. 9:1. doi: 10.1007/s13205-018-1515-5

Hillmann, B., Al-Ghalith, G. A., Shields-Cutler, R. R., Zhu, Q., Gohl, D. M., Beckman, K. B., et al. (2018). Evaluating the Information Content of Shallow Shotgun Metagenomics. mSystems 3:e0069.

Hillmann, B., Al-Ghalith, G. A., Shields-Cutler, R. R., Zhu, Q., Knight, R., and Knights, D. (2020). SHOGUN: a modular, accurate and scalable framework for microbiome quantification. Bioinformatics 36, 4088-4090. doi: 10.1093/ bioinformatics/btaa277

Jägers, C., and Roelink, H. (2019). Association of sonic hedgehog with the extracellular matrix requires its putative zinc-peptidase activity. bioRxiv[Preprint]. doi: 10.1101/2019.12.17.880039

Jia, Y., Zhao, R., and Chen, L. (2020). Similarity-based machine learning model for predicting the metabolic pathways of compounds. IEEE Access 8, 130687130696. doi: 10.1109 /access.2020.3009439

Kim, W., and Wysolmerski, J. J. (2016). Calcium-sensing receptor in breast physiology and cancer. Front. Physiol. 7:440. doi: 10.3389/fphys.2016. 00440
Kohavi, R. (1995). A study of Cross-Validation And Bootstrap For Accuracy Estimation And Model Selection in International joint Conference on artificial intelligence. Lawrence: Erlbaum Associates Ltd, 1137-1145.

Korneev, K. V., Kondakova, A. N., Sviriaeva, E. N., Mitkin, N. A., Palmigiano, A., Kruglov, A. A., et al. (2018). Hypoacylated LPS from foodborne pathogen Campylobacter jejuni induces moderate TLR4-Mediated inflammatory response in murine macrophages. Front. Cell Infect. Microbiol. 8:58. doi: 10.3389/fcimb.2018.00058

Koskela, P., Anttila, T., Bjørge, T., Brunsvig, A., Dillner, J., Hakama, M., et al. (2000). Chlamydia trachomatis infection as a risk factor for invasive cervical cancer. Int. J. Cancer 85, 35-39. doi: 10.1002/(sici)1097-0215(20000101)85: $1<35:$ :aid-ijc6>3.0.co; 2 -a

Lanfredini, S., Thapa, A., and O'neill, E. (2019). RAS in pancreatic cancer. Biochem. Soc. Trans. 47, 961-972. doi: 10.1042/BST20170521

Laurila, A. L., Anttila, T., Läärä, E., Bloigu, A., Virtamo, J., Albanes, D., et al. (1997). Serological evidence of an association between Chlamydia pneumoniae infection and lung cancer. Int. J. Cancer 74, 31-34. doi: 10.1002/(SICI)10970215(19970220)74:1<31::AID-IJC6>3.0.CO;2-1

Li, S., Chen, Z., Zhang, W., Guang, C., and Mu, W. (2019). Characterization of a D-tagatose 3-epimerase from Caballeronia fortuita and its application in rare sugar production. Int. J. Biol. Macromol. 138, 536-545. doi: 10.1016/j.ijbiomac. 2019.07.112

Liang, H., Chen, L., Zhao, X., and Zhang, X. (2020). Prediction of drug side effects with a refined negative sample selection strategy. Comp. Math. Methods Med. 2020:1573543. doi: 10.1155/2020/1573543

Liang, J. Q., Li, T., Nakatsu, G., Chen, Y.-X., Yau, T. O., Chu, E., et al. (2020). A novel faecal Lachnoclostridium marker for the non-invasive diagnosis of colorectal adenoma and cancer. Gut 69, 1248-1257. doi: 10.1136/gutjnl-2019318532

Littman, A. J., Jackson, L. A., and Vaughan, T. L. (2005). Chlamydia pneumoniae and lung cancer: epidemiologic evidence. Cancer Epidemiol. Prevent. Biomark. 14, 773-778. doi: 10.1158/1055-9965.epi-04-0599

Liu, H. A., and Setiono, R. (1998). Incremental feature selection. Appl. Intell. 9, 217-230. doi: 10.1023/A:1008363719778

Mangifesta, M., Mancabelli, L., Milani, C., Gaiani, F., De’angelis, N., De’angelis, G. L., et al. (2018). Mucosal microbiota of intestinal polyps reveals putative biomarkers of colorectal cancer. Sci. Rep. 8:13974. doi: 10.1038/s41598-01832413-2

Matthews, B. (1975). Comparison of the predicted and observed secondary structure of T4 phage lysozyme. Biochim. Biophys. Acta Prot. Struct. 405, 442-451. doi: 10.1016/0005-2795(75)90109-9

McGuire, S. (2016). "World Cancer Report 2014. Geneva: World Health Organization. doi: 10.3945/an.116.012211

Mentis, A.-F. A., Boziki, M., Grigoriadis, N., and Papavassiliou, A. G. (2019). Helicobacter pylori infection and gastric cancer biology: tempering a doubleedged sword. Cell. Mol. Life Sci. 76, 2477-2486. doi: 10.1007/s00018-01903044- 1

Nakashima, H., Nakamura, M., Yamaguchi, H., Yamanaka, N., Akiyoshi, T., Koga, K., et al. (2006). Nuclear factor- $\kappa B$ contributes to hedgehog signaling pathway activation through sonic hedgehog induction in pancreatic cancer. Cancer Res. 66, 7041-7049. doi: 10.1158/0008-5472.can-05-4588

Nolley, E., Robinson, K., Pilewski, J., Sanchez, P., D'cunha, J., and Morrell, M. (2019). Lung transplantation for patients with cystic fibrosis and achromobacter xylosoxidans in the lung allocation score era. J. Heart Lung Transpl. 38, S315-S316. doi: 10.1016/j.healun.2019.01.794

Nordlund, H. R., Hytönen, V. P., Laitinen, O. H., and Kulomaa, M. S. (2005). Novel avidin-like protein from a root nodule symbiotic bacterium. Bradyrhizobium japonicum. J. Biol. Chem. 280, 13250-13255. doi: 10.1074/jbc.m414336200

Oliveira, G. (2014). Cancer and parasitic infections: similarities and opportunities for the development of new control tools. Rev. Soc. Bras. Med. Trop. 47, 1-2. doi: 10.1590/0037-8682-0013-2014

Parida, S., and Sharma, D. (2019). The power of small changes: Comprehensive analyses of microbial dysbiosis in breast cancer. Biochim. Biophys. Acta Rev. Cancer 1871, 392-405. doi: 10.1016/j.bbcan.2019.04.001

Peng, H., Long, F., and Ding, C. (2005). Feature selection based on mutual information: criteria of max-dependency, max-relevance, and min-redundancy. IEEE Trans. Pattern Anal. Mach. Intell. 27, 1226-1238. doi: 10.1109/tpami. 2005.159 
Peters, B. A., Dominianni, C., Shapiro, J. A., Church, T. R., Wu, J., Miller, G., et al. (2016). The gut microbiota in conventional and serrated precursors of colorectal cancer. Microbiome 4:69. doi: 10.1186/s40168-016-0218-6

Poore, G. D., Kopylova, E., Zhu, Q., Carpenter, C., Fraraccio, S., Wandro, S., et al. (2020). Microbiome analyses of blood and tissues suggest cancer diagnostic approach. Nature 579, 567-574. doi: 10.1038/s41586-020-2095-1

Ramírez-Aldaba, H., Vazquez-Arenas, J., Sosa-Rodríguez, F. S., Valdez-Pérez, D., Ruiz-Baca, E., García-Meza, J. V., et al. (2017). Assessment of biofilm changes and concentration-depth profiles during arsenopyrite oxidation by Acidithiobacillus thiooxidans. Environ. Sci. Pollut. Res. 24, 20082-20092. doi: 10.1007/s11356-017-9619-8

Ray, R. B., Lagging, L. M., Meyer, K., Steele, R., and Ray, R. (1995). Transcriptional regulation of cellular and viral promoters by the hepatitis $\mathrm{C}$ virus core protein. Virus Res. 37, 209-220. doi: 10.1016/0168-1702(95)00034-n

Safavian, S. R., and Landgrebe, D. (1991). A survey of decision tree classifier methodology. IEEE Trans. Syst. Man Cyber. 21, 660-674. doi: 10.1109/21. 97458

Salk, J. J., Loubet-Senear, K., Maritschnegg, E., Valentine, C. C., Williams, L. N., Higgins, J. E., et al. (2019). Ultra-sensitive TP53 sequencing for cancer detection reveals progressive clonal selection in normal tissue over a century of human lifespan. Cell Rep. 28, 132.e133-144.e133. doi: 10.1016/j.celrep.2019. 05.109

Sang, X., Xiao, W., Zheng, H., Yang, Y., and Liu, T. (2020). HMMPred: accurate Prediction of DNA-binding proteins based on HMM Profiles and XGBoost feature selection. Comp. Math. Methods Med. 2020:1384749. doi: 10.1155/2020/ 1384749

Schultz, D., Zuhlke, D., Bernhardt, J., Francis, T. B., Albrecht, D., Hirschfeld, C., et al. (2020). An optimized metaproteomics protocol for a holistic taxonomic and functional characterization of microbial communities from marine particles. Environ. Microbiol. Rep. 12, 367-376. doi: 10.1111/1758-2229. 12842

Shams-White, M. M., Brockton, N. T., Mitrou, P., Romaguera, D., Brown, S., Bender, A., et al. (2019). Operationalizing the 2018 World Cancer Research Fund/American Institute for Cancer research (WCRF/AICR) cancer prevention recommendations: a standardized scoring system. Nutrients 11:1572. doi: 10. 3390/nu11071572

Shibata, T., Lieblong, B. J., Sasagawa, T., and Nakagawa, M. (2019). The promise of combining cancer vaccine and checkpoint blockade for treating HPV-related cancer. Cancer Treat. Rev. 78, 8-16. doi: 10.1016/j.ctrv.2019.07.001

Smith, J. S., Bosetti, C., Munoz, N., Herrero, R., Bosch, F. X., Eluf-Neto, J., et al. (2004). Chlamydia trachomatis and invasive cervical cancer: a pooled analysis of the IARC multicentric case-control study. Int. J. Cancer 111, 431-439. doi: $10.1002 /$ ijc. 20257

Srivastava, R., Rolyan, H., Xie, Y., Li, N., Bhat, N., Hong, L., et al. (2019). TCF7L2 (Transcription Factor 7-Like 2) Regulation of GATA6 (GATA-Binding Protein 6)-Dependent and -Independent Vascular Smooth Muscle Cell Plasticity and Intimal Hyperplasia. Arterioscl. Thromb. Vasc. Biol. 39, 250-262. doi: 10.1161/ atvbaha.118.311830

Sun, L., Liu, H., Zhang, L., and Meng, J. (2015). IncRScan-SVM: a tool for predicting long non-coding RNAs using support vector machine. PLoS One 10:e0139654. doi: 10.1371/journal.pone.0139654.g007

Thackray, V. G. (2019). Sex, microbes, and polycystic ovary syndrome. Trends Endocrinol. Metab. 30, 54-65. doi: 10.1016/j.tem.2018.11.001

Vanhoutte, G., Van De Wiel, M., Wouters, K., Sels, M., Bartolomeeussen, L., De Keersmaecker, S., et al. (2016). Cachexia in cancer: what is in the definition? BMJ Open Gastroenterol 3:e000097. doi: 10.1136/bmjgast-2016000097

Vivarelli, S., Salemi, R., Candido, S., Falzone, L., Santagati, M., Stefani, S., et al. (2019). Gut microbiota and cancer: from pathogenesis to therapy. Cancers 11:38. doi: $10.3390 /$ cancers 11010038
Wang, Q., Yang, S., Wang, K., and Sun, S.-Y. (2019). MET inhibitors for targeted therapy of EGFR TKI-resistant lung cancer. J. Hematol. Oncol. 12:63. doi: 10.1186/s13045-019-0759-9

Wani, B., Aziz, S. A., Ganaie, M. A., and Mir, M. H. (2017). Metabolic syndrome and breast cancer risk. Indian J. Med. Paediatr. Oncol. 38, 434-439. doi: 10. 4103/ijmpo.ijmpo_168_16

Wei, L., Xing, P., Shi, G., Ji, Z. L., and Zou, Q. (2017). Fast prediction of protein methylation sites using a sequence-based feature selectxion technique. IEEE/ACM Trans Comput Biol Bioinform. 16, 1264-1273. doi: 10.1109/TCBB. 2017.2670558

Weng, H., Zeng, X. T., Li, S., Kwong, J. S., Liu, T. Z., and Wang, X. H. (2016). Tea consumption and risk of bladder cancer: a dose-response meta-analysis. Front. Physiol. 7:693. doi: 10.3389/fphys.2016.00693

Witten, I. H., and Frank, E. (2005). Data Mining: Practical Machine Learning Tools and Techniques. Burlington, MA: Morgan Kaufmann Pub.

Wood, D. E., Lu, J., and Langmead, B. (2019). Improved metagenomic analysis with Kraken 2. Genome Biol. 20:257. doi: 10.1186/s13059-019-1891-0

Wood, D. E., and Salzberg, S. L. (2014). Kraken: ultrafast metagenomic sequence classification using exact alignments. Genome Biol. 15:R46. doi: 10.1186/gb2014-15-3-r46

Wu, J., Yang, J., Ding, J., Guo, X., Zhu, X. Q., and Zheng, Y. (2018). Exosomes in virus-associated cancer. Cancer Lett. 438, 44-51. doi: 10.1016/j.canlet.2018. 09.018

Yamaguchi, F., Takata, M., Kamitori, K., Nonaka, M., Dong, Y., Sui, L., et al. (2008). Rare sugar D-allose induces specific up-regulation of TXNIP and subsequent G1 cell cycle arrest in hepatocellular carcinoma cells by stabilization of p27kip1. Int. J. Oncol. 32, 377-385. doi: 10.3892/ijo.32.2.377

Yang, H., Qu, L., Ma, H., Chen, L., Liu, W., Liu, C., et al. (2010). Mycoplasma hyorhinisinfection in gastric carcinoma and its effects on the malignant phenotypes of gastric cancer cells. BMC Gastroenterol. 10:132. doi: 10.1186/ 1471-230X-10-132

Yu, Z., Song, G., Liu, J., Wang, J., Zhang, P., and Chen, K. (2018). Beneficial effects of extracellular polysaccharide from Rhizopus nigricans on the intestinal immunity of colorectal cancer mice. Int. J. Biol. Macromol. 115, 718-726. doi: 10.1016/j.ijbiomac.2018.04.128

Zhao, X., Chen, L., and Lu, J. (2018). A similarity-based method for prediction of drug side effects with heterogeneous information. Math. Biosci. 306, 136-144. doi: $10.1016 /$ j.mbs.2018.09.010

Zhou, J.-P., Chen, L., and Guo, Z.-H. (2020a). iATC-NRAKEL: an efficient multilabel classifier for recognizing anatomical therapeutic chemical classes of drugs. Bioinformatics 36, 1391-1396. doi: 10.1093/bioinformatics/btz757

Zhou, J.-P., Chen, L., Wang, T., and Liu, M. (2020b). iATC-FRAKEL: A simple multi-label web-server for recognizing anatomical therapeutic chemical classes of drugs with their fingerprints only. Bioinformatics 36, 3568-3569. doi: 10. 1093/bioinformatics/btaa166

Zhu, Y.-Y., Zhou, L.-P., Zhang, Q., Hu, Y., and Fang, Z.-X. (2007). Isolation of Mycoplasma penetrans from blood and tissue specimens of patients with cervical cancer. Chin. J. Zoon. 23:537.

Conflict of Interest: The authors declare that the research was conducted in the absence of any commercial or financial relationships that could be construed as a potential conflict of interest.

Copyright (c) 2020 Chen, Li, Zeng, Zhang, Liu, Li, Huang and Cai. This is an openaccess article distributed under the terms of the Creative Commons Attribution License (CC BY). The use, distribution or reproduction in other forums is permitted, provided the original author(s) and the copyright owner(s) are credited and that the original publication in this journal is cited, in accordance with accepted academic practice. No use, distribution or reproduction is permitted which does not comply with these terms. 